\title{
Avant-propos
}

\section{Filière cunicole française et systèmes d'élevage}

La filière cunicole française a connu des évolutions techniques majeures et une structuration continue au cours des dernières décennies. Elles ont été permises, entre autres, par des efforts de recherche publique en nutrition, génétique, reproduction, éthologie et santé animale. En introduction de ce dossier spécial «Actualités en recherche cunicole» nous présentons de façon très synthétique les systèmes d'élevage et la filière cunicole française en rappelant au préalable les particularités physiologiques du lapin qui ont des conséquences fortes sur son mode d'élevage.

\section{1 / Rappels sur la biologie du lapin et conséquences sur les systèmes de production}

\section{1 / Reproduction et relations mères-jeunes}

La lapine est une femelle polytoque ayant une durée de gestation de 31 jours, dont l'ovulation est induite par l'accouplement. Contrairement à de nombreux mammifères, elle ne présente pas d'anœestrus post-partum, mais est à l'inverse très réceptive dans les heures qui suivent la parturition. Un pourcentage élevé de femelles peut également être fécondé tout au long de la lactation. L'éleveur peut donc choisir lui-même le rythme de reproduction qu'il utilise dans son élevage.

Pendant très longtemps, les éleveurs ont pratiqué une conduite à la semaine, voire à la journée : chaque semaine à jour fixe ou bien chaque jour, les femelles aptes à la reproduction sont présentées au mâle pour l'accouplement.

Au début des années 90, la filière cunicole a pris de nouvelles orientations avec le développement de la conduite en bandes (avec cycles de reproduction synchronisés) associée à l'insémination artificielle. Ce système améliore l'organisation du travail et de l'enlèvement des animaux, et il offre la possibilité de réaliser un vide sanitaire entre chaque bande. Mais pour être rentable, il nécessite une bonne maîtrise de la fertilité des femelles. Ce sujet fait l'objet de travaux de recherche (cf. article de Theau-Clément).

Au moment de la naissance, les lapereaux sont sourds et aveugles et leurs fonctions motrices sont peu développées. Leur alimentation est exclusivement lactée dans les premières semaines de vie. Leur survie dépend donc de leur capacité à localiser rapidement la tétine maternelle lors de l'unique allaitement quotidien. Ce challenge, qui sollicite fortement le système olfactif, intervient dans un contexte de compétition intense au sein de la portée. Le développement et les facteurs de régulation du comportement du jeune lapereau avant sevrage font l'objet de l'article de Coureaud $\boldsymbol{e t}$ al.

En conditions naturelles, le sevrage intervient de manière progressive et à un âge qui fluctue en fonction de l'offre alimentaire ( 2 à 6 semaines). En élevage, le sevrage est pratiqué entre 4 et 5 semaines d'âge, et les animaux destinés à la consommation sont abattus entre 10 et 11 semaines, ils ont alors un poids vif moyen de 2,4 $\mathrm{kg}$ (tableau 1 ).

\section{2 / Nutrition}

Le lapin possède également une physiologie digestive particulière. Comme le cheval, c'est un monogastrique herbivore. Par contre, il a pour spécificité de pratiquer la cæcotrophie. Le lapin excrète deux types de fèces : les fèces dures et les cæcotrophes. Ces derniers correspondent à l'excrétion d'une partie du contenu caecal après un passage rapide dans le colon. Seuls les cæcotrophes sont consommés par l'animal, ce qui permet de valoriser des protéines et des vitamines d'origine bactérienne.

En élevage rationnel, le lapin est nourri avec un aliment complet granulé, contenant l'ensemble des nutriments nécessaires à ses besoins. Par rapport à d'autres monogastriques (porc, poulet) le lapin a un besoin supplémentaire en fibres. L'apport de fibres alimentaires doit être relativement important (16 à 20\% de lignocellulose) pour un fonctionnement optimal du tube digestif, et réduire ainsi les risques de troubles digestifs (diarrhées). Les fibres alimentaires sont partiellement hydrolysées et fermentées par les microorganismes hébergés dans le cæcum et le colon. Les relations entre écosystème cæcal, nutrition et santé digestive du lapin font l'objet de l'article de Gidenne et al.
Tableau 1. Performances moyennes en 2006 des élevages conduits en bandes qui utilisent une gestion technico-économique en France (Lebas 2007).

\begin{tabular}{lc}
\hline Nombre d'ateliers & $\mathbf{1 0 8 9}$ \\
\hline Nombre moyen de femelles en production & 495 \\
Renouvellement des femelles (\%) & 113 \\
Taux de mortalité des femelles (\%) & 29,1 \\
Fertilité (MB/IA, \%) & \\
Intervalle entre 2 MB (j) & 52,8 \\
Nombre MB/femelle/an & 6,9 \\
Nombre total de lapereaux nés/MB & 10,1 \\
Nombre de lapereaux sevrés/MB & 8,1 \\
Nombre de lapins produits/MB & 7,4 \\
Taux mortalité naissance - vente/nés totaux (\%) & 26,7 \\
Nb lapereaux vendus/femelle/an & 50,7 \\
Poids vif moyen à la vente (kg) & 2,45 \\
Age moyen à la vente (j) & 74,3 \\
Indice de consommation d'élevage & 3,58 \\
Marge/coût alimentaire/femelle/an (€) & 118,8 \\
Marge/coût alimentaire/kg lapin produit (€) & 0,95 \\
\hline
\end{tabular}

$\mathrm{MB}$ : mise bas 
En élevage rationnel, les femelles sont généralement alimentées à volonté tandis qu'au cours de leur croissance les lapereaux peuvent être soumis à des phases de restriction alimentaire afin de réduire la fréquence des troubles digestifs. L'achat d'aliment granulé complet et équilibré entraîne une situation de dépendance de l'atelier vis-à-vis du secteur de l'alimentation animale et rompt une partie du lien qui rattache l'atelier de production animale au sol de l'exploitation. Cela fait partie des pratiques qui sont considérées comme limitantes lorsque l'on réalise une analyse de la durabilité des conditions de production du lapin (cf. article de Fortun-Lamothe).

\section{2 / La filière cunicole française}

\section{1 / La production de lapin}

Avec $80000 \mathrm{~T}$ de carcasse produites chaque année, la cuniculture française se situe au 4ème rang mondial, après la Chine $(450000 \mathrm{~T})$, l'Italie $(225000 \mathrm{~T})$ et l'Espagne $(108000 \mathrm{~T})$. Depuis l'adhésion des nouveaux pays membres, l'Union Européenne à 25 représente une production de $515000 \mathrm{~T}$. C'est donc la première zone productrice du monde, talonnée par la Chine. Les quatre pays cités ci-dessus représentent près de $72 \%$ de la production mondiale estimée à environ 1,2 million de tonnes selon la FAO (progression de $+13,5 \%$ par rapport à 2000, due surtout à la Chine). Pour la France, les échanges globaux de lapins (import/export) sont légèrement excédentaires $(\approx 1500 \mathrm{~T})$.

Cependant, la production cunicole française reste encore aujourd'hui difficile à apprécier avec précision. Ainsi en 2000, le recensement général agricole comptabilisait 4880 exploitations cunicoles avec plus de 20 femelles reproductrices. Mais ce chiffre diminue régulièrement, en raison d'une rationalisation importante de la filière qui entraîne une hausse de la taille moyenne des élevages professionnels, désormais d'environ 450 femelles (=1 UTH). Ces élevages sont concentrés pour $75 \%$ dans 5 grandes régions en France, dont 65\% dans le grand ouest (Bretagne, Pays de la Loire, Poitou-Charentes). La fabrication d'aliments composés pour le lapin est d'environ $500000 \mathrm{~T}$. Bien qu'il reste difficile de quantifier exactement le nombre d'emplois générés dans l'amont et l'aval de la filière, on peut estimer que chaque élevage génère de 3 à 4 emplois induits, ce qui correspondrait pour les 3500 élevages spécialisés à plus de 10000 emplois induits.

La consommation française de viande de lapin est estimée à $1,2 \mathrm{~kg} / \mathrm{hab} / \mathrm{an}$. Comme pour les autres viandes, cette consommation tend à se réduire, sachant que la vente de viande de lapin est assurée à $74 \%$ par les grandes et moyennes surfaces.

\section{2 / Le contexte technico-économique}

L'évolution de la production s'est accompagnée d'une spécialisation des élevages. Les exploitations cunicoles françaises sont confrontées aux exigences de compétitivité inscrites dans le fonctionnement des marchés. Sur le long terme, ceci se traduit par la recherche constante d'une réduction des coûts de production. Ceci a été possible grâce à l'amélioration de l'efficacité des élevages (productivité de l'atelier maternité, coût alimentaire...). Toutefois ces tendances sont actuellement mises à mal du fait de l'augmentation spectaculaire du prix de l'aliment en 2007 et 2008, en relation avec la conjoncture internationale sur le prix des céréales. De plus, la maîtrise du statut sanitaire reste délicate en élevage cunicole. Les troubles digestifs restent très fréquents chez les jeunes lapereaux au cours de la période qui suit le sevrage. Sur le troupeau des femelles reproductrices, on observe fréquemment des problèmes respiratoires chroniques (pasteurelles). L'article de Licois et Marlier fait le point sur les recherches en pathologie cunicole.

La viande de lapin ne présente aucun défaut majeur de qualité, et sa valeur nutritionnelle a fait l'objet d'une synthèse récente dans cette revue (Combes 2004). Actuellement, le principal signe officiel de qualité développé par la filière est la production sous certification de conformité (1/3 des élevages). La production «label rouge» reste marginale en raison de coûts élevés de production (et de difficulté de maîtrise sanitaire) tandis que la production «biologique» reste «confidentielle» pour les mêmes raisons. Les produits découpés progressent aux dépens du produit classique présenté sous forme de «carcasse entière».

Les qualités nutritionnelles et diététiques de la viande de lapin sont reconnues riches en protéines et pauvres lipides (peu de cholestérol), riches en minéraux mais pauvres en sodium, source d'oméga 3 avec un faible ratio $\omega 6 / \omega 3$ (de 4 à 6 ).

\section{3 / Conduite de l'élevage et performances technico-économiques}

Le modèle naisseur-engraisseur est présent pour la quasi-totalité des élevages. Les exploitations cunicoles restent en grande majorité familiales (1 à 2 UTH). Les lapines présentes dans les élevages de production sont issues d'élevages de multiplication, eux-mêmes approvisionnés par des élevages de sélection (structure pyramidale de la sélection). Les femelles de production sont donc très majoritairement issues de croisements (Néo-Zélandais Blanc x Californien), dont les lignées proviennent généralement des schémas de sélection de l'INRA. Les travaux de recherches en génétique portent aujourd'hui sur de nouveaux caractères d'intérêt zootechnique (cf. article de Garreau $\boldsymbol{e t} \boldsymbol{a l}$ ).

L'ITAVI centralise chaque année les résultats technico-économiques des éleveurs de lapins de chair en production organisée dans le cadre de programmes d'appui technique. Ces données permettent de suivre l'évolution des performances technico-économiques des élevages français (Lebas 2007). Les performances moyennes des élevages pratiquant la conduite en bande est reportée dans le tableau 1.

L. Fortun-Lamothe, T. Gidenne

\section{Références}

Combes S., 2004. Valeur nutritionnelle de la viande de lapin. INRA Prod. Anim., 17, 373-383.

Lebas F., 2007. Productivité des élevages cunicoles professionnels en 2006. Résultats de RENALAP et RENACEB. Cuniculture Magazine, $34,31-39$. 